optimize therapy. In 2 cases, there was a rupture of the shockwave balloon, and this was believed to be due to inadequate balloon preparation, the procedure continued with a replacement shockwave balloon without any complication. Baseline characteristics are described in table 1.

Discussion During this study, 2 episodes of hypotension were recorded during the procedures, one recovered immediately with the challenge of normal saline, the second patient who was admitted as ACS (NSTEMI), had a heavy calcified left main disease that required bifurcation stenting, Intra-aortic balloon was required for 24 hours. The patient was discharged

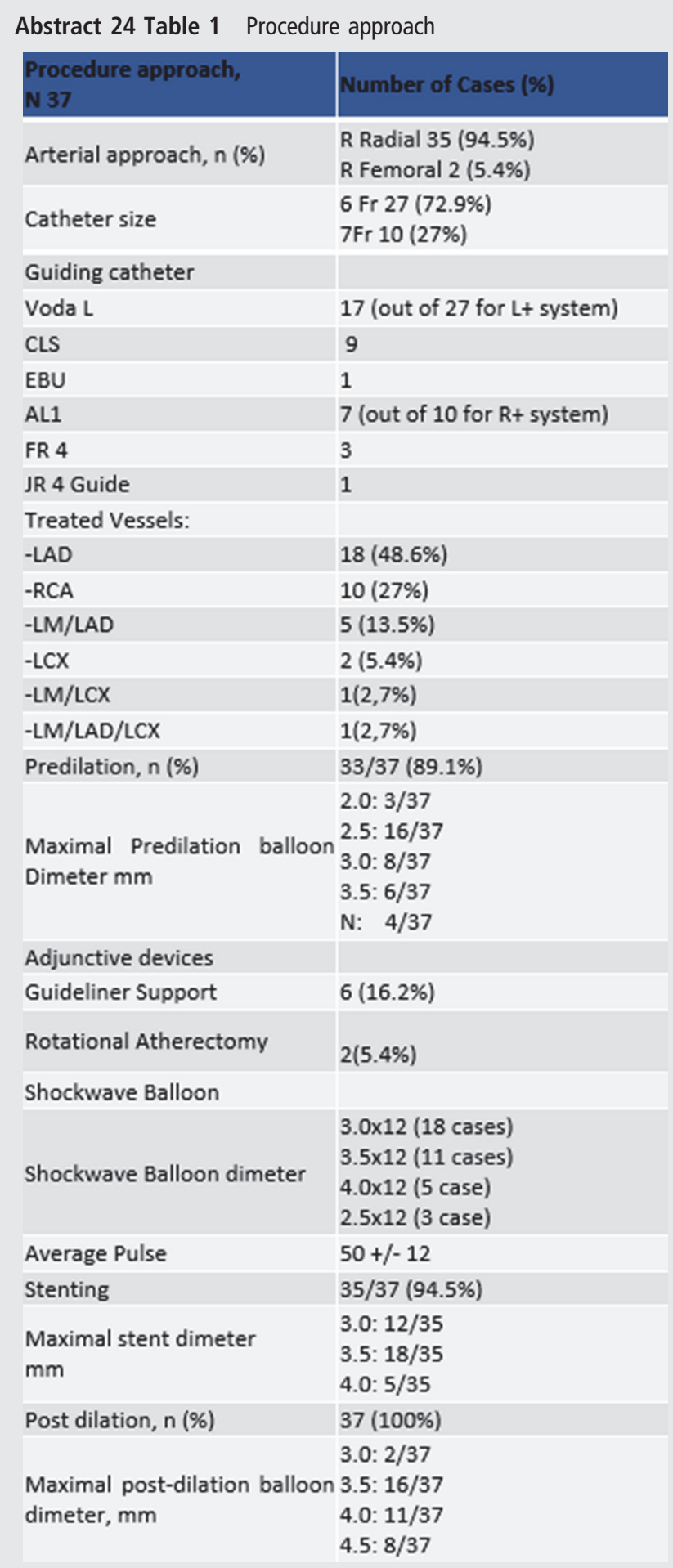

home after 7 days. Two years' follow-up showed 4 cases required to repeat coronary angiography; One case atypical chest pain with the non-obstructive disease, the second case had new lesions in different coronary arteries. The third; with prior history of CABG and stent to the native RCA with ISR had IVUS guided NC ballooning. The last one also prior to CABG, had severe ISR of his native LM/LCX even after highpressure balloon and DEB treatment. The patient was referred for Laser ablation \pm Re-do CABG. Unfortunately, due to comorbidities this patient was not fit for these procedures and passed away after two months.

Conclusion Initial experience with intravascular lithotripsy has demonstrated good angiographic outcomes without significant complications even after stent implantation. S-IVL has a minimal learning curve and has been quickly adopted as a safe and successful alternative to Rotablation. If shockwaves fail Rotablation still remains an option (2 of our cases). Patients with a previous history of CABG had a lower success rate reflective of the chronicity and complexity of the disease.

\section{CT CORONARY ANGIOGRAPHY AND COVID-19: INPATIENT USE IN ACUTE CHEST PAIN SERVICE}

M Cronin, P Wheen, R Armstrong, R Kumar, A McMahon, M White, N Sheehy, G McMahon, R Murphy. St. Lukes Hospital Kilkenny, Ireland

\subsection{6/heartjnl-2021-ICS.25}

Introduction CT coronary angiography (CTCA) is a well-validated clinical tool in the evaluation of chest pain. In January 2020 a care pathway was agreed between the cardiology, radiology and emergency departments in our facility to implement a strategy of CTCA as a first line inpatient examination for selected patients with chest pain of low to moderate risk of significant coronary artery disease. Impact on length of stay and clinical outcomes including subsequent invasive angiography and revascularisation were evaluated, including major adverse cardiac events at 30 days.

Methods Study design was retrospective. Patients referred for cardiology review between October 2019 and May 2020 with chest pain and/or dyspnoea were broken into three cohorts: a baseline cohort, a cohort with increased CTCA availability and a cohort with increased CTCA availability, but after the national lockdown due to COVID-19 in Ireland. These patients were identified via the Hospital Inpatient Enquiry dataset and correlated with chart review. Statistical analysis

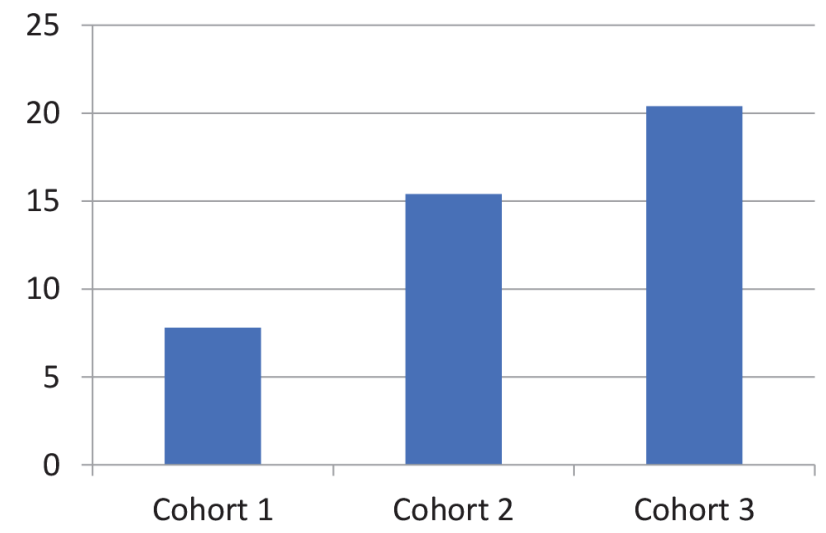

Abstract 25 Figure 1 Number of CTCA (\% of performance in overall cohort) 
was via SPSS. Ethical approval was provided by the research and ethics committee in St. James Hospital, Dublin.

Results Between October 2019 and May 2020513 patients $(35.3 \%$ female) presented between cohorts $1 \quad(n=179), 2$ $(n=182)$, and $3(n=153)$. Overall population characteristics, investigations and outcomes are available in table 1 . This same information is available for those who underwent CTCA in table 2. Daily rate of ED referral to cardiology increased from 2.32 to 2.46 and 2.73 patients. Overall length of stay between cohorts 1 and 3 decreased from 2.66 days median to 1.71 days $(p=0.014) .73$ inpatient CTCA were performed over the 8 month period, increasing from $7.8 \%$ overall in cohort $1 \%$ to $20.4 \%$ in cohort 3 (see figure 1). A history of

Abstract 25 Table 1 Overall characteristics

\begin{tabular}{|c|c|c|c|}
\hline & $\begin{array}{l}14 / 10 / 19-30 / 12 / \\
19\end{array}$ & $13 / 1 / 20-27 / 3 / 20$ & $27 / 3 / 20-22 / 5 / 20$ \\
\hline Total & 179 & 182 & 153 \\
\hline Female & $59(33 \%)$ & $71(39.9 \%)$ & $51(33.3 \%)$ \\
\hline \multirow[t]{2}{*}{$\begin{array}{l}\text { Presenting } \\
\text { complaint }\end{array}$} & $\begin{array}{l}153 \text { Chest pain } \\
(85.5 \%)\end{array}$ & $\begin{array}{l}151 \text { Chest Pain (83\%) } \\
6 \text { Dyspnoea (3\%) }\end{array}$ & $\begin{array}{l}131 \text { Chest Pain } \\
(85.6 \%)\end{array}$ \\
\hline & $\begin{array}{l}26 \text { Dyspnoea } \\
(14.5 \%)\end{array}$ & $\begin{array}{l}25 \text { Chest pain/Dyspnoea } \\
(14 \%)\end{array}$ & $\begin{array}{l}8 \text { Dyspnoea }(5.2 \%) \\
14 \text { Chest pain/ } \\
\text { Dyspnoea }(9.2 \%)\end{array}$ \\
\hline Age & $62.6(55.7-71.8)$ & $59(50-70)$ & $59(50-70)$ \\
\hline $\begin{array}{l}\text { Length of stay } \\
\text { (days) }\end{array}$ & $2.66(0.96-4.92)$ & $2.16(1.2-5.1)$ & $1.71(1-3.3)$ \\
\hline Family history & $84(46.9 \%)$ & $41(22.5 \%)$ & $40(26.1 \%)$ \\
\hline Hypertension & $100(55.9 \%)$ & $85(46.7 \%)$ & $60(39.2 \%)$ \\
\hline Diabetes Mellitus & $31(17.3 \%)$ & $20(11 \%)$ & $12(7.8 \%)$ \\
\hline \multirow[t]{3}{*}{ Smoker } & 49 Current (27.4\%) & 46 Current $(25.3 \%)$ & 38 Current (25\%) \\
\hline & $66 \operatorname{Ex}(36.9 \%)$ & 61 Ex $(33.5 \%)$ & $53 \operatorname{Ex}(34.9 \%)$ \\
\hline & 64 Never $(35.7 \%)$ & 75 Never $(41.2 \%)$ & 62 Never $(40.1 \%)$ \\
\hline Dyslipidaemia & $94(52.5 \%)$ & $97(53.3 \%)$ & $71(46.4 \%)$ \\
\hline $\begin{array}{l}\text { History of } \\
\text { cardiovascular/ } \\
\text { cerebrovascular } \\
\text { disease }\end{array}$ & $74(41.3 \%)$ & $67(36.8 \%)$ & $50(32.7 \%)$ \\
\hline $\begin{array}{l}\text { Systolic blood } \\
\text { pressure }\end{array}$ & $126(110-114)$ & $136(121-153)$ & $144(125-156)$ \\
\hline $\begin{array}{l}\text { Diastolic blood } \\
\text { pressure }\end{array}$ & $74(66-81.5)$ & $81(72-89)$ & $82(74-89)$ \\
\hline Heart rate & $70(64-79)$ & $76(66-86)$ & $72(65-84)$ \\
\hline Weight (kilogram) & $78(69.9-90.9)^{*}$ & $80(70-89.4)^{*}$ & $\begin{array}{l}80.5(73.8-93.5) \\
\text { * }\end{array}$ \\
\hline $\begin{array}{l}\text { New ECG } \\
\text { ischaemia }\end{array}$ & $63(35.2 \%)$ & $36(19.8 \%)$ & $22(14.3 \%)$ \\
\hline Troponin-T (ng/L) & $15(0-44)$ & $0(0-21.5)$ & $0(0-20)$ \\
\hline $\begin{array}{l}\text { eGFR }(\mathrm{ml} / \mathrm{min} / \\
1.73 \mathrm{~m} 2)\end{array}$ & $84(67-99)$ & $90(75-107)$ & $89(72-103)$ \\
\hline CTCA amount & $14(7.8 \%)$ & $28(15.4 \%)$ & $31(20.3 \%)$ \\
\hline $\begin{array}{l}\text { Exercise stress } \\
\text { test }\end{array}$ & $43(24 \%)$ & $47(25.8 \%)$ & $0(0 \%)$ \\
\hline $\begin{array}{l}\text { Invasive Coronary } \\
\text { Angiography }\end{array}$ & $82(45.8 \%)$ & $71(39 \%)$ & $52(33.9 \%)$ \\
\hline $\begin{array}{l}\text { Revascularisation } \\
\text { performed }\end{array}$ & $53(29.6 \%)$ & $29(15.9 \%)$ & $25(16.3 \%)$ \\
\hline Death at 1 month & $6(3.4 \%)$ & $1(0.5 \%)$ & $2(1.3 \%)$ \\
\hline $\begin{array}{l}\text { Myocardial } \\
\text { infarction at } 1 \\
\text { month }\end{array}$ & 0 & 1 & 1 \\
\hline
\end{tabular}

\begin{tabular}{|c|c|c|c|}
\hline \multirow{18}{*}{$\begin{array}{l}\text { Outpatient } \\
\text { functional/ } \\
\text { anatomical } \\
\text { imaging }\end{array}$} & Total $23(12.8 \%) 9$ & Total $28(15.4 \%) 10$ & Total $26(16.9 \%)$ \\
\hline & Invasive coronary & Stress perfusion cardiac & $11 \mathrm{CT}$ coronary \\
\hline & angiogram (39\%) & magnetic resonance & angiography \\
\hline & 4 Stress perfusion & imaging (35.7\%) & $(42.3 \%)$ \\
\hline & cardiac magnetic & 6 Invasive coronary & 9 Invasive coronary \\
\hline & resonance imaging & angiogram (21.4\%) & angiogram (34.6\%) \\
\hline & $(17.4 \%)$ & $5 \mathrm{CT}$ coronary & 4 Stress perfusion \\
\hline & 4 Dobutamine & angiography (18\%) & cardiac magnetic \\
\hline & stress & 4 Single-photo emission & resonance imaging \\
\hline & echocardiography & computed tomography & $(15.4 \%)$ \\
\hline & $(17.4 \%)$ & $(14 \%)$ & 1 Dobutamine \\
\hline & 3 Single-photo & 3 Dobutamine stress & stress \\
\hline & emission computed & echocardiography & echocardiography \\
\hline & tomography & $(10.9 \%)$ & $(3.85 \%)$ \\
\hline & $(13.1 \%)$ & & 1 Single-photo \\
\hline & $3 \mathrm{CT}$ coronary & & emission computed \\
\hline & angiography & & tomography \\
\hline & $(13.1 \%)$ & & $(3.85 \%)$ \\
\hline
\end{tabular}

Weight: Not available $=36$ in cohort 1, 39 in cohort 2, 50 in cohort 3

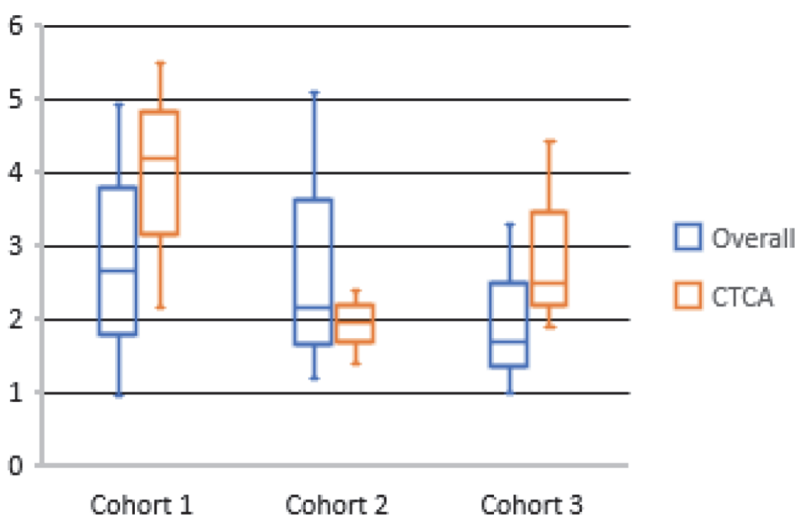

Abstract 25 Figure 2 Overall/CTCA length of stay median/IQR (days)

Abstract 25 Table 2 CTCA cohort characteristics

\begin{tabular}{|c|c|c|c|c|}
\hline & $\begin{array}{l}14 / 10 / 19- \\
30 / 12 / 19\end{array}$ & $\begin{array}{l}13 / 1 / 20- \\
27 / 3 / 20\end{array}$ & $\begin{array}{l}27 / 3 / 20- \\
22 / 5 / 20\end{array}$ & $\begin{array}{l}14 / 10 / 19- \\
27 / 3 / 20\end{array}$ \\
\hline Total & $14(7.8 \%)$ & $28(15.4 \%)$ & $31(20.4 \%)$ & 73 \\
\hline Female & $5(35.7 \%)$ & $14(50 \%)$ & $12(38.7 \%)$ & $31(42.5 \%)$ \\
\hline Age & $\begin{array}{l}61.1(57.8- \\
67.4)\end{array}$ & $\begin{array}{l}50.5(44.8- \\
56.5)\end{array}$ & $\begin{array}{l}54(50- \\
60.5)\end{array}$ & $55(49-60)$ \\
\hline Length of stay (days) & $\begin{array}{l}4.2(2.16- \\
5.5)\end{array}$ & $\begin{array}{l}1.98(1.4- \\
2.4)\end{array}$ & $\begin{array}{l}2.5(1.9- \\
4.5)\end{array}$ & $2.2(1.7-4.3)$ \\
\hline Family History & $5(36 \%)$ & $8(28.6 \%)$ & $10(32.3 \%)$ & $23(31.5 \%)$ \\
\hline Hypertension & $6(42.9 \%)$ & $4(14.3 \%)$ & $10(32.3 \%)$ & $20(27.4 \%)$ \\
\hline Diabetes Mellitus & $2(14.3 \%)$ & $1(3.6 \%)$ & $0(0 \%)$ & $3(4.1 \%)$ \\
\hline Smoker & $\begin{array}{l}\text { Current }=4 \\
(28.6 \%) \\
\text { Ex }=6 \\
(42.8 \%) \\
\text { Never }=4 \\
(28.6 \%)\end{array}$ & $\begin{array}{l}\text { Current = } 9 \\
(32.1 \%) \\
\text { Ex }=7(25 \%) \\
\text { Never }=12 \\
(42.9 \%)\end{array}$ & $\begin{array}{l}\text { Current }= \\
9(29 \%) \\
\text { Ex }=11 \\
(35.5 \%) \\
\text { Never }=11 \\
(35.5 \%)\end{array}$ & $\begin{array}{l}\text { Current }=22 \\
(30.1 \%) \\
\text { Ex }=24 \\
(32.9 \%) \\
\text { Never }=27 \\
(37 \%)\end{array}$ \\
\hline Dyslipidaemia & $5(35.7 \%)$ & $12(42.9 \%)$ & $10(32.3 \%)$ & $27(37 \%)$ \\
\hline $\begin{array}{l}\text { History of cardiovascular/ } \\
\text { cerebrovascular disease }\end{array}$ & $0(0 \%)$ & $3(10.7 \%)$ & $0(0 \%)$ & $3(4.1 \%)$ \\
\hline Weight (kilogram) & $\begin{array}{l}74(64- \\
80.8)^{*}\end{array}$ & $\begin{array}{l}72(65.8- \\
78.8)^{*}\end{array}$ & $\begin{array}{l}80.2(74.5 \\
-88.2)^{*}\end{array}$ & $\begin{array}{l}76(69- \\
83.5)^{*}\end{array}$ \\
\hline
\end{tabular}




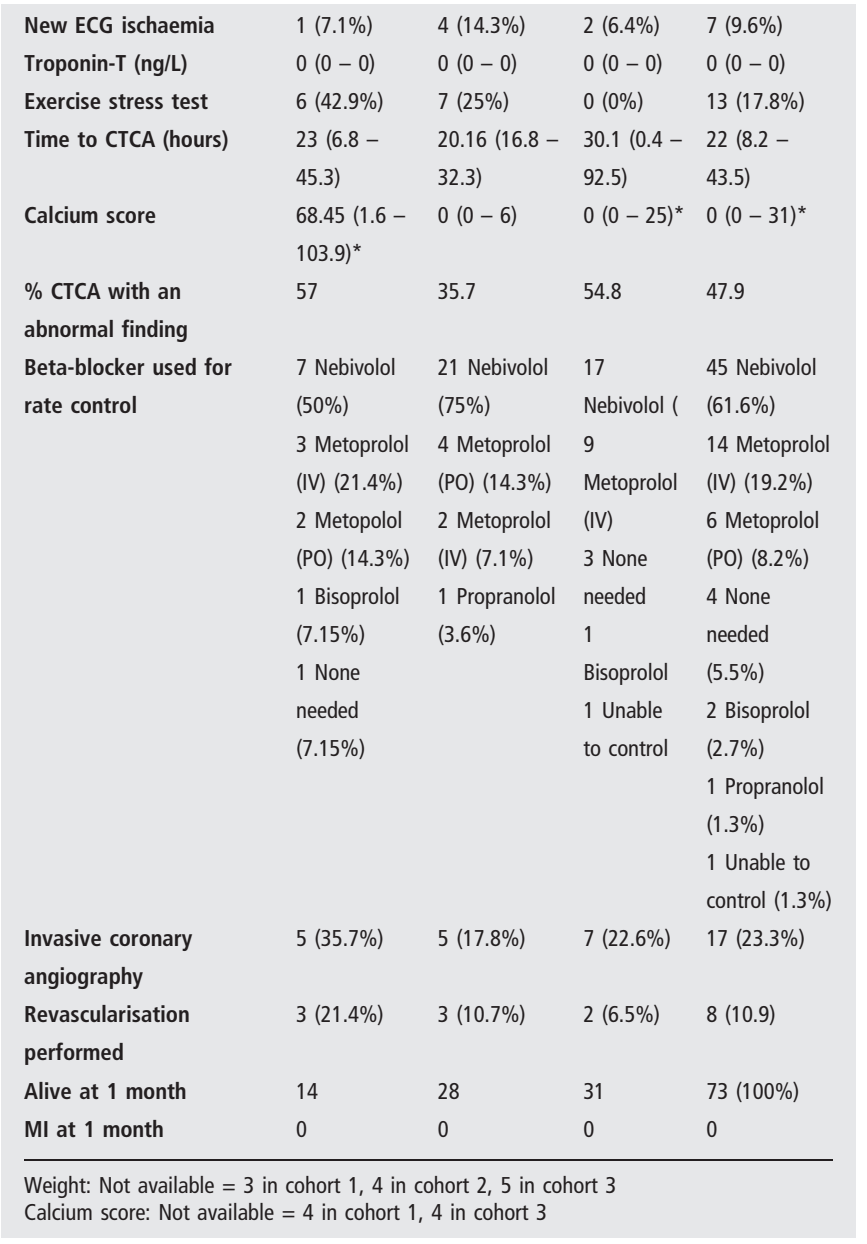

cerebrovascular/cardiovascular disease was significantly associated with CTCA use $(\mathrm{p}=0.00097)$ in cohort 1 . Age $(\mathrm{p}=$ $0.0017)$, hypertension $(p=0.001)$ and history of cerebrovascular/cardiovascular disease $(\mathrm{p}=0.0015)$ were associated with CTCA use in cohort 2. Diabetes $(p=0.037)$ and history of cerebrovascular/cardiovascular disease $(p=0.000246)$ were associated with CTCA use in cohort 3. Overall length of stay for the patients undergoing CTCA decreased from a median of 4.2 days in cohort 1 to 2.5 days in cohort 3 (see figure 2), with no increase in 30 days adverse outcomes. Invasive coronary angiography rate dropped from $45.8 \%$ to $39 \%$ in cohort 2 , and to $34.2 \%$ in cohort $3(\mathrm{p}=0.0286)$. Revascularization was required in $29.6 \%$ in cohort $1,15.9 \%$ in cohort 2 , and $16.3 \%$ in cohort $3(\mathrm{p}=0.00448)$.

Conclusions This study demonstrated an increase in inpatient CTCA provision. The doubling of proportion of patients investigated by CTCA from $7.8 \%$ to $15.4 \%$ was associated with a reduction in length of stay from 4.2 days to 1.98 days. The improved efficiency in processing these intermediate rather than high risk patients was maintained during the COVID-19 pandemic, with a continued lower length of stay. A corresponding reduction in the rates of invasive coronary angiography and revascularization was seen, without an increase in 30-day major adverse cardiac events. We envisage our findings to be referenced when encouraging the development of CTCA use within national healthcare systems, particularly in light of the increasing trend towards shortening length of hospital admissions within the context of an international pandemic.

\section{CARDIAC DEVICE REMOTE MONITORING - FREQUENCY OF CLINICALLY IMPORTANT EVENT IN RELATION TO INTERVENTION}

${ }^{1}$ LR Hamel, ${ }^{2} \mathrm{~A}$ Mittal, ${ }^{2} \mathrm{~A}$ Rehman, ${ }^{2} \mathrm{~J} \mathrm{O}^{\prime} \mathrm{Neill},{ }^{2} \mathrm{~J}$ Galvin, ${ }^{2} \mathrm{E}$ Keelan, ${ }^{2} \mathrm{R}$ O'Mahony, ${ }^{2} \mathrm{~T} \mathrm{O}^{\prime}$ Neill. ${ }^{1}$ Royal College of Surgeons in Ireland - Medical University of Bahrain, Bahrain; ${ }^{2}$ Connolly Hospital Blanchardstown, Dublin, Ireland

\subsection{6/heartjnl-2021-ICS.26}

Introduction Remote Cardiac Device Monitoring (RCDM) has been an advancing technology in the field for many years. Assessing the efficacy, safety and logistics of the RCDM ensures patient safety. This study set out to examine the time from clinically relevant events to an intervention in view of the potential risk of either missed events or delay in discovery of such events. There are no national standardized operating procedures concerning RCDM in Ireland, which puts responsibility on cardiac teams to ensure in-house protocols and stringent strategies are in place to provide best practice and patient safety.

\section{Avg Response Time in Days (All Companies)}

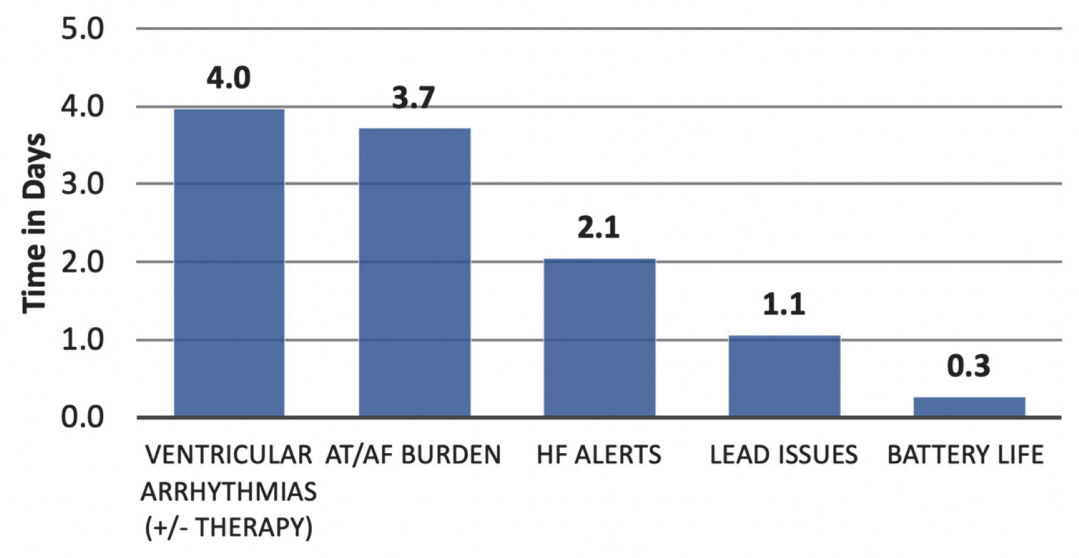

Type of Alert

Abstract 26 Figure 1 Total number of transmissions to average time to intervention in days in all three companies 